\title{
Multicarrier DS/SFH-CDMA Systems
}

\author{
Jiangzhou Wang, Senior Member, IEEE, and Hu Huang
}

\begin{abstract}
In this paper, multicarrier direct-sequence/slow-frequency-hopping (MC DS/SFH) code-division multiple-access (CDMA) systems are proposed, in which multiple carriers are modulated by the same DS waveform and hopped in frequency according to a random hopping pattern. The receiver dehops the received signal with the same pattern, provides $R A K E$ receivers for each carrier, and combines the outputs with a maximal ratio combiner (MRC). The performance of the proposed system is investigated over a frequency-selective Rayleigh-fading channel and compared to that of the MC DS-CDMA systems. It is shown that for the same diversity order, the MC DS/SFH-CDMA systems are superior in reducing multiple-access interference (MAI) while preserving the good capability of narrow-band interference suppression, when the system parameters are selected properly.
\end{abstract}

Index Terms-Code-division multiple access (CDMA), multicarrier (MC) systems, narrow-band interference suppression.

\section{INTRODUCTION}

$\mathbf{I}$ $\mathrm{N}$ ORDER to increase the spectral efficiency of wireless communications, it has been proposed to overlay a spread spectrum (SS) system on existing narrow-band systems [1], [2]. The SS signal must be transmitted at a much lower power level to avoid adversely affecting the narrow-band systems, which in turn may raise significant interference to the SS systems. A pure direct-sequence (DS) system without suppression filters usually does not work well in this situation.

Multicarrier (MC) code-division multiple-access (CDMA) systems are ingenious in suppressing narrow-band interference [3]-[7], where the entire frequency band is subdivided into multiple frequency slots (subchannels) of equal width. A replica of a DS signal is transmitted in each subchannel and the outputs of all subchannels are combined in a way that the distorted one contributes less to the final decision. Since the interference concerned is narrow-band (i.e., it does not overlay all of the subchannels), the decision can be made on the basis of the unaffected subchannels and interference suppression is achieved. In addition, the MC systems require a lower chip rate, which reduces the implementation complexity.

An adaptive algorithm has been proposed [4] to exploit the correlation between the received signals on different carriers to cancel multiple-access interference (MAI) and make the MC DS system robust to the near-far problems. However, the algorithm usually needs 10 to 20 symbol intervals to converge. An alternative resort is the frequency hopping $(\mathrm{FH})$ technique [8], [9], in which the signal occupies only one subchannel at any interval and hops in frequency. By reducing the number of simultaneous

Manuscript received April 11, 2000; revised August 24, 2001.

$\mathrm{J}$. Wang is with the Department of Electrical and Electronic Engineering, The University of Hong Kong, Hong Kong (e-mail: jwang@eee.hku.hk).

H. Huang is with the Department of Electrical and Computer Engineering, University of Maryland, College Park, MD 20742 USA.

Digital Object Identifier 10.1109/TVT.2002.801758 cochannel users, FH systems have a natural guard against the near-far problem. However, their performance in the presence of narrow-band interference is not satisfactory without coding [10].

The systems combining these two techniques, called multicarrier direct-sequence/slow-frequency-hopping CDMA (MC DS/SFH-CDMA) systems, in which multiple subchannels are used to transmit the same information during each hop period, are expected to show good capability in combating both narrow-band interference and MAI. In this paper, the uplink performance of the proposed system is studied in a multipath Rayleigh-fading channel and compared to that of MC DS-CDMA systems, given the same information rate, the same diversity order, and same system bandwidth. One possible application of this proposed scheme is ultra-wide-band communications with bandwidth as large as $3 \mathrm{GHz}$.

The remainder of the paper is organized as follows. In Section II, the system models are described. In Section III, the performance of the MC DS/SFH-CDMA systems in terms of average bit-error rate (BER) is analyzed. Numerical results are given in Section IV. Finally, the conclusions are presented in Section V.

\section{SYSTEM MODELS}

In an MC DS/SFH system, the total system bandwidth $B_{T}$ is divided into $S=M Q$ contiguous subchannels, where $M$ is the number of simultaneous carriers at any instant and $Q$ is the number of available hopping frequencies. Each subchannel has a bandwidth $B_{s}=B_{T} / S$, and the central frequency of the $s$ th subchannel is denoted as $f_{s}(s=q+m Q, q=1, \ldots, Q$, and $m=0, \ldots, M-1)$. The transmitter for the $k$ th user is shown in Fig. 1, where $b_{k}(t)$ is the data sequence with bit duration $T$ and bit rate $R_{b}=1 / T, a_{k}(t)$ is the random signature sequence with chip duration $T_{c}=T / N$, where $N$ is the processing gain of one subchannel. The bit and chip waveforms are all rectangular, and the spread signal is band-limited by its null-to-null bandwidth $2 / T_{c}$, which is equal to $B_{s}$. The total processing gain is $N_{T}=B_{T} /(2 / T)=S N$. The hopping pattern $f_{k}(t)$ takes values randomly from $\left\{f_{1}, f_{2}, \ldots, f_{Q}\right\}$ at any hop interval, and the frequency separation between the nearest two carriers $B_{H}=Q B_{s}$. Therefore, if two users take different hopping frequencies, there is no overlap between their transmitted signals in frequency.

The transmitted signal of the $k$ th user can be written as

$$
S_{k}(t)=\operatorname{Re}\left[\sqrt{2 P} \sum_{m=0}^{M-1} C_{k m}(t)\right]
$$

where $P$ is the average transmitted power of each carrier and

$C_{k m}(t)=b_{k}(t) a_{k}(t) \exp \left\{j 2 \pi\left[f_{k}(t)+m B_{H}\right] t+j \theta_{k, m}\right\}$

where $\theta_{k, m}$ is the random phase introduced. 


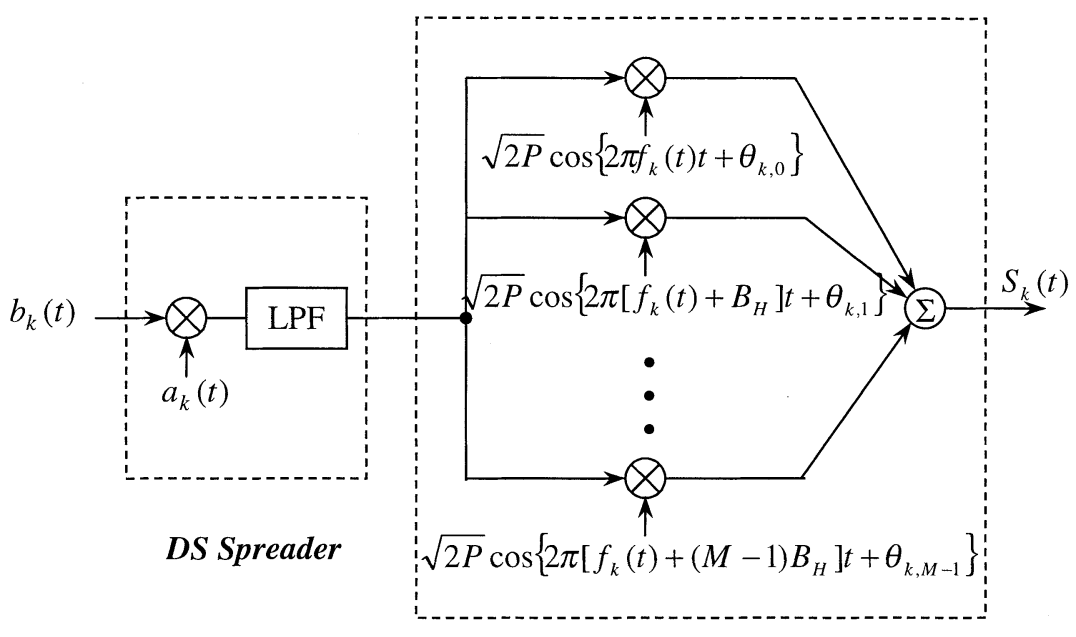

Multicarrier modulator

Fig. 1. The transmitter structure of the MC DS/SFH system.

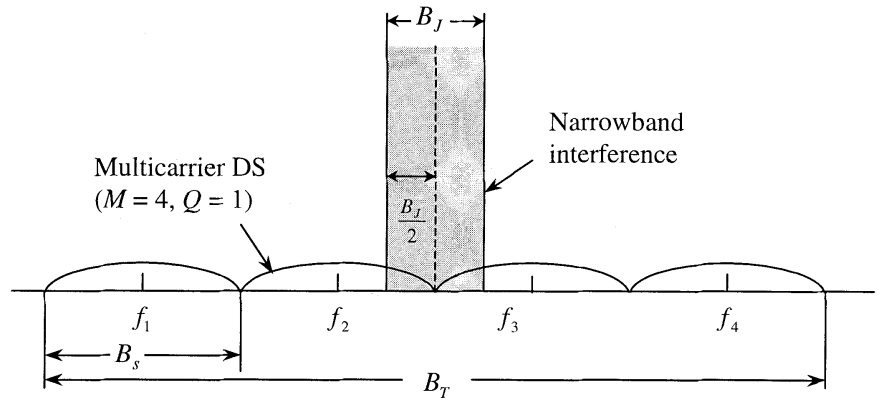

(a)

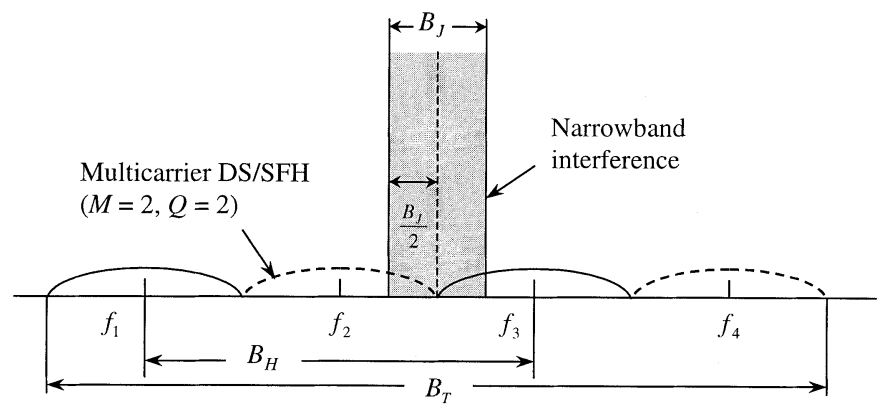

(b)

Fig. 2. Power spectrum densities of transmitted signals and the narrow-band interference.

Note that $M=S$ and $Q=1$ corresponds to the MC DS system proposed in [3]. The power spectrum densities of the transmitted signals of the MC DS and the MC DS/SFH system are shown in Fig. 2, where the number of total subchannels $S=$ 4. Each carrier has the same transmitted power in both the MC DS and MC DS/SFH systems. Therefore, the total transmitted power of the MC DS/SFH system is only $1 / Q$ of that of the MC DS system, due to a smaller number of carriers. At the receiver end, multiple antennas are needed to ensure the same order of diversity and the same total received energy per bit for the MC DS/SFH system.

The mobile radio channel is assumed to be frequency selective fading with delay spread of $T_{m}$. The $s$ th subchannel for the $k$ th user and the $v$ th antenna can be well approximated by a tapped-delay-line model [10] with complex low-pass equivalent impulse response given by

$$
h_{k}^{(s, v)}(t)=\sum_{l=0}^{L-1} \beta_{k l}^{(s, v)} \exp \left[j \mu_{k l}^{(s, v)}\right] \delta\left(t-l T_{c}-\tau_{k}\right)
$$

where $L$ is the number of resolvable paths and given by

$$
L=\left\lfloor T_{m} / T_{c}\right\rfloor+1=\left\lfloor T_{m} B_{T} /(2 S)\right\rfloor+1 .
$$

Letting $L_{T}=\left\lfloor T_{m} B_{T} / 2\right\rfloor+1$ denote the number of resolvable paths (also the order of diversity available) in a pure DS system, one obtains

$$
L= \begin{cases}\left\lfloor\left(L_{T}-1\right) / S\right\rfloor+1 \approx L_{T} / S, & S \leq L_{T} \\ 1, & S>L_{T} .\end{cases}
$$

The fading gains $\left\{\beta_{k l}^{(s, v)}\right\}$ are assumed to be identically Rayleigh-distributed and mutually independent, provided that $B_{s}$ is wider than the channel coherence bandwidth $\left(1 / T_{m}\right)$. It is assumed that the delay power profile is uniform and perfect power control is achieved, i.e., $E\left[\left(\beta_{k l}^{(s, v)}\right)^{2}\right]=2 \rho$ for all $l$ and $k$. The phases $\left\{\mu_{k l}^{(s, v)}\right\}$ are assumed to be uniformly and independently distributed in $[-\pi, \pi) . \tau_{k}$ is the relative delay of the first path signal of the $k$ th user and is randomly distributed in $\left[0, T-(L-1) T_{c}\right)$.

The narrow-band interference is characterized as band-limited white Gaussian noise with the double-sided power spectral density $\eta_{J} / 2$ and bandwidth $B_{J}$ (see Fig. 2). For simple analysis, it is assumed that the interference is located at the center of the system bandwidth. The ratio of the interference bandwidth to the system bandwidth is defined as

$$
p=\frac{B_{J}}{B_{T}}
$$

and the ratio of the interference power to the signal power is defined as

$$
w=\frac{P_{J}}{P_{s}}=\frac{\eta_{J} B_{J}}{E_{b} / T},
$$

where $E_{b}$ is the average total received signal energy per bit.

Considering the case when $B_{J} \leq B_{s}$ and $S$ is even, the interference is limited in the two middle subchannels and causes 


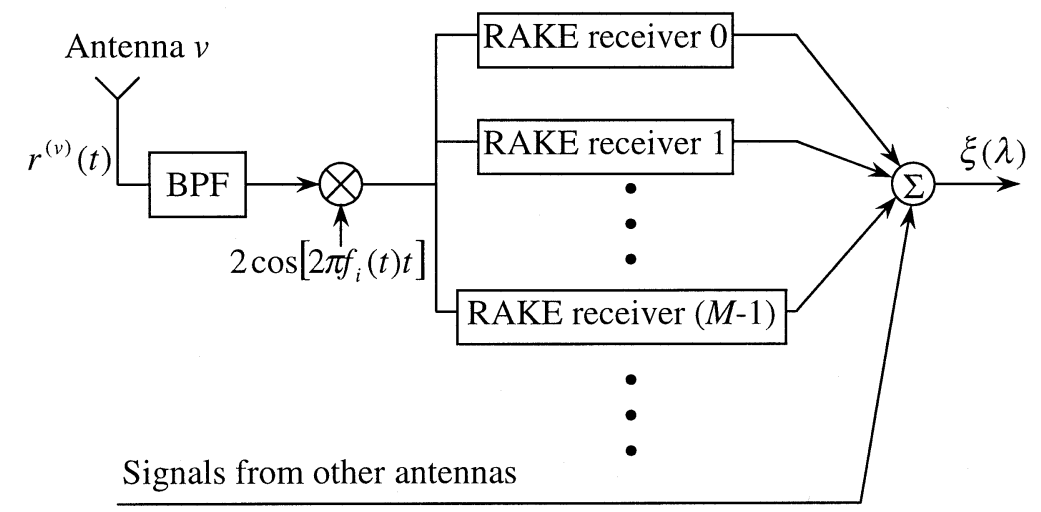

(a)

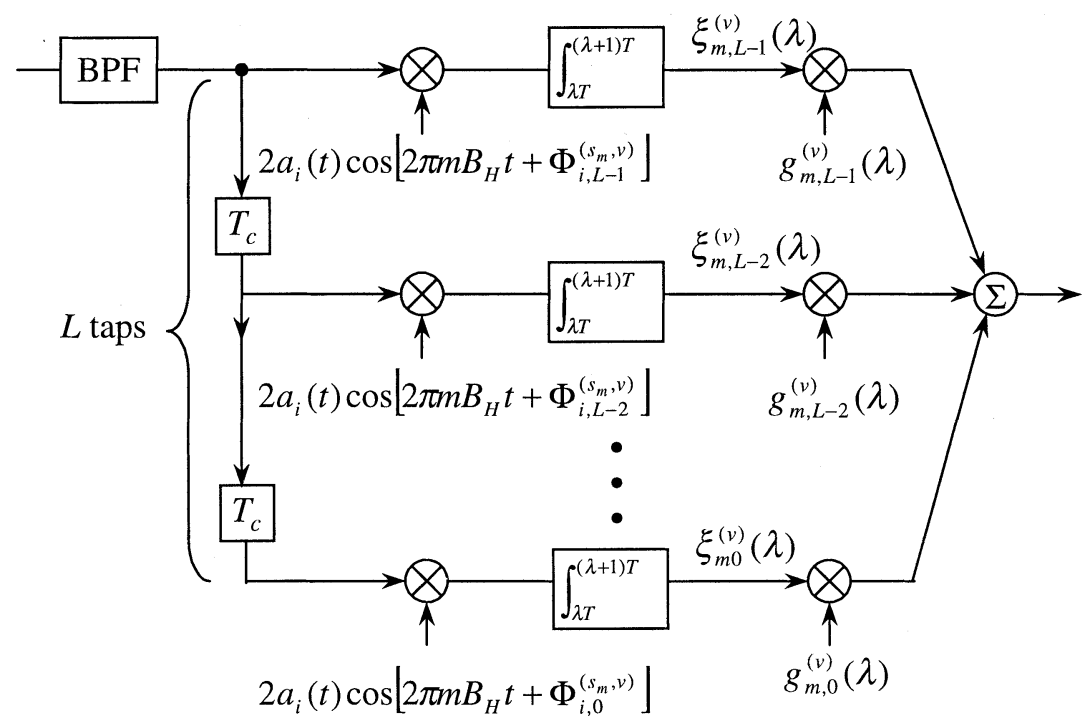

(b)

Fig. 3. The receiver structures (a) MC DS/SFH system and (b) detailed block diagram of RAKE receiver \#m for user $i$.

no impairment to others. Assuming that each subchannel employs an ideal bandpass filter, the interference can be viewed as two independent interferers with bandwidth $B_{J} / 2$, one in each of the two subchannels. The interference in the sth subchannel received by the $v$ th antenna $J^{(s, v)}(t)$ can then be written as

$$
\begin{aligned}
J^{(s, v)}(t)=\Delta(s) & \cdot \operatorname{Re}\left\{\left[J_{I}^{(v)}(t)+j J_{Q}^{(v)}(t)\right]\right. \\
\cdot & \left.\exp \left\{j 2 \pi\left[f_{s} \pm\left(B_{s} / 2-B_{J} / 4\right)\right] t\right\}\right\}
\end{aligned}
$$

where

$$
\Delta(s)=[\delta(s, S / 2)+\delta(s, S / 2+1)]
$$

\pm takes + for $s=S / 2$ and - for $s=S / 2+1$, respectively. $\delta(x, y)=1$ and 0 for $x=y$ and $x \neq y$, respectively. Both $J_{I}^{(v)}(t)$ and $J_{Q}^{(v)}(t)$ are independent lowpass processes with

$$
E\left[\left(J_{I}^{(v)}(t)\right)^{2}\right]=E\left[\left(J_{Q}^{(v)}(t)\right)^{2}\right]=P_{J} / 2 .
$$

The autocorrelation function of $J^{(s, v)}(t)$ is given by

$$
\begin{aligned}
R_{J}^{(s, v)}(\tau)=\Delta(s) P_{J} / 2 & \cdot \operatorname{sinc}\left(\pi B_{J} \tau / 2\right) \\
\cdot & \cdot \cos \left\{2 \pi\left[f_{s} \pm\left(B_{s} / 2-B_{J} / 4\right)\right] \tau\right\}
\end{aligned}
$$

where $\operatorname{sinc}(x)=\sin (x) / x$.
The received signal by antenna $v$ can be written as [the local clock is synchronized with the $(L-1)$ th path signal]

$r^{(v)}(t)$

$$
\begin{aligned}
= & \operatorname{Re}\left[\sqrt{2 P} \sum_{k=1}^{K} \sum_{m=0}^{M-1} C_{k m}(t) * h_{k}^{\left(s_{k m}, v\right)}\left[t+(L-1) T_{c}\right]\right] \\
+ & n^{(v)}(t)+\sum_{s=1}^{S} J^{(s, v)}(t) \\
= & \operatorname{Re}\left\{\sqrt{2 P} \sum_{k=1}^{K} \sum_{m=0}^{M-1} \sum_{l=0}^{L-1} \beta_{k l}^{\left(s_{k m}, v\right)}\right. \\
& \left.\cdot \exp \left[j \mu_{k l}^{\left(s_{k m}, v\right)}\right] C_{k m}\left[t-l T_{c}-\tau_{k}+(L-1) T_{c}\right]\right\} \\
+ & n^{(v)}(t)+\sum_{s=1}^{S} J^{(s, v)}(t)
\end{aligned}
$$

where $K$ stands for the number of users, $s_{k m}$ is the subchannel occupied by the $m$ th carrier of the $k$ th user. $n^{(v)}(t)$ is the additive white Gaussian noise (AWGN) with double-sided spectral density $N_{0} / 2$. The receiver for the reference user (the $i$ th user) is shown in Fig. 3(a). The received signal is first dehopped according to the hopping pattern of the reference user, and the 
output is fed to $M$ RAKE receivers. The outputs of all RAKE receivers are combined to obtain the final decision variable.

The detailed structure of the $m$ th RAKE receiver is shown in Fig. 3(b). A single delay line is employed through which the input signal is passed. The $l$ th resolvable path signal is collected by the $l$ th tap, which correlates the delayed input signal with the spreading sequence. The correlator outputs are combined by a maximal ratio combiner. For simplicity, we do not consider the cross correlations between the delayed code sequences. Consequently, a RAKE receiver with perfect estimates of the tap gains is equivalent to a maximal ratio combiner with $L$ th-order diversity. When $V$ antennas are employed and $M$ RAKE receivers for each of them, the whole receiver is equivalent to a maximal ratio combiner with diversity order equal to $V M L$. Note that $M L=L_{T} / Q$ when $S \leq L_{T}$. If we choose $V=Q$, all systems have the same diversity order equal to $L_{T}$.

\section{Performance AnAlysis}

In this paper, we aim to compare the capabilities of different systems in suppressing MAI and narrow-band interference. Perfect tracking of all path signals of the reference user is assumed, as well as perfect power control and channel estimation. Therefore, the performance presented in what follows should be considered as the lower bounds of the practical systems.

In the presence of narrow-band interference, the performance of an SFH system depends on the instantaneous value of its hopping frequency. Conditioned on $f_{i}(\lambda T)=f_{q}$, the output of the correlator of the $l$ th tap of the $m$ th RAKE receiver in the $\lambda$ th symbol interval is given by

$$
\begin{aligned}
\xi_{m l}^{(v)}(\lambda)= & \int_{\lambda T}^{(\lambda+1) T} 2 r^{(v)}\left[t-(L-1-l) T_{c}\right] \\
& \cdot \cos \left\{2 \pi f_{q}\left[t-(L-1-l) T_{c}\right]\right\} \\
& \cdot 2 a_{i}(t) \cos \left[2 \pi m B_{H} t+\Phi_{i, l}^{\left(s_{m}, v\right)}\right] d t
\end{aligned}
$$

where $s_{m}=q+m Q$ denotes the subchannel occupied by the $m$ th carrier of the $i$ th user's signal $\left(f_{s_{m}}=f_{q}+m B_{H}\right)$ and

$$
\Phi_{i, l}^{\left(s_{m}, v\right)}=\theta_{i, m}+\mu_{i l}^{\left(s_{m}, v\right)}+2 \pi f_{q}(L-1-l) T_{c} .
$$

Neglecting the high-frequency terms, (9) can be written as

$$
\xi_{m l}^{(v)}(\lambda)=D_{m l}^{(v)}+N_{m l}^{(v)}+J_{m l}^{(v)}+P_{m l}^{(v)}+I_{m l}^{(v)}
$$

where

$$
D_{m l}^{(v)}=\sqrt{2 P} T \beta_{i l}^{\left(s_{m}, v\right)} b_{i}^{(\lambda)}
$$

is the desired signal component, where $b_{i}^{(\lambda)}$ stands for the $\lambda$ th bit of the reference user with $b_{i}^{(\lambda)}=1$ or -1 .

$N_{m l}^{(v)}$ is a Gaussian random variable with zero mean and variance equal to $N_{0} T$.

$J_{m l}^{(v)}$ is due to the narrow-band interference in the $s_{m}$ th subchannel and can be written as

$$
\begin{aligned}
J_{m l}^{(v)}= & \int_{\lambda T}^{(\lambda+1) T} J^{\left(s_{m}, v\right)}\left[t-(L-1-l) T_{c}\right] 2 \\
& \cdot \cos \left[2 \pi f_{q}\left[t-(L-1-l) T_{c}\right]\right] \\
& \cdot 2 a_{i}(t) \cos \left[2 \pi m B_{H} t+\Phi_{i, l}^{\left(s_{m}, v\right)}\right] d t .
\end{aligned}
$$

Since $J^{\left(s_{m}, v\right)}(t)$ is a Gaussian process, $J_{m l}^{(v)}$ is also a zero-mean Gaussian random variable. Its variance $\sigma_{J}^{2}\left(s_{m}\right)$, conditioned on $s_{m}$, is derived in Appendix A as

$$
\sigma_{J}^{2}\left(s_{m}\right)=\sigma_{J}^{2}(q+m Q)=\Delta(q+m Q) \frac{P_{J} T^{2}}{N} \hat{\sigma}_{J}^{2}
$$

where $\Delta(s)$ is given by $(6 \mathrm{~b}), P_{J}$ is the interference power, and $\hat{\sigma}_{J}^{2}$ is given by

$\hat{\sigma}_{J}^{2}=\int_{0}^{1} \sin c(\pi p S \tau) \cos [2 \pi(1-p S / 2) \tau](1-\tau)(1-\tau / N) d \tau$

where $p$ is given by (4) and $S$ is the number of subchannels.

$P_{m l}^{(v)}\left(l^{\prime} \neq l\right)$ is the total multipath interference term from the reference user. Since the number of resolvable paths in MC systems has been greatly reduced, as the result of lower chip rate, $P_{m l}^{(v)}$ is insignificant and can be neglected for simple analysis.

$I_{m l}^{(v)}=\sum_{k=1}^{K_{h}} \sum_{l^{\prime}=0}^{L-1} I_{k l^{\prime}}^{(m, v)}$ is the total MAI term, where $K_{h}$ is the number of nonreference users that collide with the reference user in frequency $\left(f_{k}(\lambda T)=f_{q}\right)$. Assuming the hopping patterns of different users are mutually independent, $K_{h}$ has a binomial distribution with probability density function (pdf) given by

$$
P_{K_{h}}\left(K_{h}\right)=\left(\begin{array}{c}
K-1 \\
K_{h}
\end{array}\right)\left(P_{h}\right)^{K_{h}}\left(1-P_{h}\right)^{K-K_{h}-1}
$$

where $P_{h}$ is the probability that a nonreference user hits the reference user and approximates to $1 / Q$ for $\mathrm{SFH}$ [8]. Note that (13) also applies to the pure DS and the MC DS systems, i.e., $P_{K_{h}}\left(K_{h}\right)=\delta\left(K_{h}, K-1\right)$, due to $P_{h}=1$ for these two systems.

$I_{k l^{\prime}}^{(m, v)}$ is due to the $l^{\prime}$ th path signal of the $k$ th user $(k \neq i)$ and is given by

$$
\begin{aligned}
I_{k l^{\prime}}^{(m, v)}= & \sqrt{2 P} \beta_{k l^{\prime}}^{\left(s_{m}, v\right)} \cos \left[\Phi_{k, l^{\prime}}^{\left(s_{m}, v\right)}-\Phi_{i, l}^{\left(s_{m}, v\right)}\right] \\
& \cdot \int_{\lambda T}^{(\lambda+1) T} b_{k}\left(t-\Gamma_{l^{\prime} l}^{k}\right) a_{k}\left(t-\Gamma_{l^{\prime} l}^{k}\right) a_{i}(t) d t \\
= & \sqrt{2 P} \beta_{k l^{\prime}}^{\left(s_{m}, v\right)} \cos \left[\Phi_{k, l^{\prime}}^{\left(s_{m}, v\right)}-\Phi_{i, l}^{\left(s_{m}, v\right)}\right] \\
& \cdot\left[b_{k}^{(\lambda-1)} R_{k i}\left(\Gamma_{l^{\prime} l}^{k}\right)+b_{k}^{(\lambda)} \hat{R}_{k i}\left(\Gamma_{l^{\prime} l}^{k}\right)\right]
\end{aligned}
$$

where

$$
\Phi_{k, l^{\prime}}^{\left(s_{m}, v\right)}=\theta_{k, m}+\mu_{k l^{\prime}}^{\left(s_{m}, v\right)}-2 \pi f_{s_{m}} \Gamma_{l^{\prime} l}^{k}+2 \pi f_{q}(L-1-l) T_{c}
$$

and $\Gamma_{l^{\prime} l}^{k}=\tau_{k}+\left\{l^{\prime}-l\right) T_{c} . b_{k}^{(\lambda-1)}$ and $b_{k}^{(\lambda)}$ stand for the $(\lambda-1)$ th and the $\lambda$ th bit of the $k$ th user, respectively, which are assumed to be uncorrelated and take values randomly from $\{+1,-1\}$. The partial cross-correlation functions $R_{k i}(\tau)$ and $\hat{R}_{k i}(\tau)$ are defined as

$$
\begin{aligned}
& R_{k i}(\tau)=\int_{0}^{\tau} a_{k}(t-\tau) a_{i}(t) d t \\
& \hat{R}_{k i}(\tau)=\int_{\tau}^{T} a_{k}(t-\tau) a_{i}(t) d t .
\end{aligned}
$$

It is easy to prove that $\left\{I_{k l^{\prime}}^{(m, v)}\right\}$ are identically distributed but uncorrelated for different $k$ and $l$. When $K_{h} L$ is large, one can 
use the Gaussian approximation [11], and treat $I_{m l}^{(v)}$ as a random Gaussian variable with variance, conditioned on $K_{h}$, given by

$$
\begin{aligned}
\sigma_{I}^{2}\left(K_{h}\right)= & \sum_{k=1}^{K_{h}} \sum_{l^{\prime}=0}^{L-1} P \cdot E\left[\left(\beta_{k l^{\prime}}^{\left(s_{m}, v\right)}\right)^{2}\right] \\
& \cdot E\left[R_{k i}^{2}\left(\Gamma_{l^{\prime} l}^{k}\right)+\hat{R}_{k i}^{2}\left(\Gamma_{l^{\prime} l}^{k}\right)\right] \\
= & 4 K_{h} L P \rho T^{2} /(3 N)
\end{aligned}
$$

where

$$
E\left[R_{k i}^{2}\left(\Gamma_{l^{\prime} l}^{k}\right)\right]=E\left[\hat{R}_{k i}^{2}\left(\Gamma_{l^{\prime} l}^{k}\right)\right]=T^{2} /(3 N) .
$$

The signal-to-noise ratio (SNR) of $\xi_{m l}^{(v)}(\lambda)$, conditioned on $\beta_{i l}^{\left(s_{m}, v\right)}, K_{h}$, and $q$ is then defined as

$$
\begin{aligned}
&\left.\gamma_{m l}^{(v)}\right|_{\beta_{i l}^{\left(s_{m}, v\right)}, K_{h}, q} E^{2}\left[\xi_{m l}^{(v)}(\lambda) \mid \beta_{i l}^{\left(s_{m}, v\right)}\right] \\
&= 2 \operatorname{var}\left[\xi_{m l}^{(v)}(\lambda) \mid K_{h}, q\right] \\
&= \frac{2 P T^{2}\left(\beta_{i l}^{\left(s_{m}, v\right)}\right)^{2}}{2\left[\sigma_{I}^{2}\left(K_{h}\right)+\sigma_{J}^{2}(q+m Q)+\sigma_{N}^{2}\right]} \\
&= \frac{\left(\beta_{i l}^{\left(s_{m}, v\right)}\right)^{2}}{2 \rho}\left[2 K_{h} L /(3 N)+\Delta(q+m Q) \frac{P_{J} T}{E_{c}} \frac{\hat{\sigma}_{J}^{2}}{N}\right. \\
&\left.\quad+\left(E_{c} / N_{0}\right)^{-1}\right]^{-1}
\end{aligned}
$$

where $E_{c}=2 \rho P T$ is the average received signal energy per diversity branch.

By definition, $\left\{N_{m l}^{(v)}\right\}$ are independent and identically distributed (i.i.d.). It is also easy to verify that $\left\{I_{m l}^{(v)}\right\}$ and $\left\{J_{m l}^{(v)}\right\}$ are mutually uncorrelated. Therefore, $\left\{\xi_{m l}^{(v)}(\lambda)\right\}$ are uncorrelated for different $m, l$, and $v$. To maximize the SNR of the final decision variable, each $\xi_{m l}^{(v)}(\lambda)$ is weighted by a tap gain [3]

$$
g_{m, l}^{(v)}(\lambda)=\frac{E\left[b_{i}^{(\lambda)} \xi_{m l}^{(v)}(\lambda) \mid \beta_{i l}^{\left(s_{m}, v\right)}\right]}{\operatorname{var}\left[\xi_{m l}^{(v)}(\lambda) \mid K_{h}, q\right]}
$$

and the final decision variable is given by

$$
\xi(\lambda)=\sum_{v=1}^{V} \sum_{m=0}^{M-1} \sum_{l=0}^{L-1} g_{m, l}^{(v)}(\lambda) \xi_{m l}^{(v)}(\lambda) .
$$

The instantaneous SNR of $\xi(\lambda)$, conditioned on $\left\{\beta_{i l}^{\left(s_{m}, v\right)}\right\}, K_{h}$, and $q$ is given by

$$
\begin{aligned}
\left.\gamma_{b}\right|_{\left\{\beta_{i l}^{\left(s_{m}, v\right)}\right\}, K_{h}, q} & \frac{E^{2}\left[\xi(\lambda) \mid\left\{\beta_{i l}^{\left(s_{m}, v\right)}\right\}\right]}{2 \operatorname{var}\left[\xi(\lambda) \mid K_{h}, q\right]} \\
= & \left.\sum_{v=1}^{V} \sum_{m=0}^{M-1} \sum_{l=0}^{L-1} \gamma_{m l}^{(v)}\right|_{\beta_{i l}^{\left(s_{m}, v\right)}, K_{h}, q} \\
= & \sum_{v=1}^{V} \sum_{m=0}^{M-1} \sum_{l=0}^{L-1} \frac{\left(\beta_{i l}^{\left(s_{m} v\right)}\right)^{2}}{2 \rho}\left\{2 K_{h} L /(3 N)\right. \\
& \left.+D\left[\Delta(q+m Q) w \hat{\sigma}_{J}^{2} / N+\left(E_{b} / N_{0}\right)^{-1}\right]\right\}^{-1}
\end{aligned}
$$

where $D=V M L$ is the total order of diversity and $E_{b}=$ $D E_{c}$ is the total received signal energy per bit, and $w$ is the interference power to the signal power ratio defined in (5).

The conditional BER takes the simple form

$$
P_{e}\left(\gamma_{b}\right)=\frac{1}{\sqrt{2 \pi}} \int_{\sqrt{2 \gamma_{b}}}^{\infty} e^{-x^{2} / 2} d x .
$$

To get the final average BER $P_{e}$, we first find the BER $\left.P_{e}\right|_{K_{h}, q}$, conditioned on $K_{h}$ and $q$, which can be obtained by averaging $P_{e}\left(\gamma_{b}\right)$ over the statistics of $\left\{\beta_{i l}^{\left(s_{m}, v\right)}\right\}$. The detailed derivation of $\left.P_{e}\right|_{K_{h}, q}$ is shown in Appendix B. Since all hopping frequencies appear with equal probability, the final average BER is given by

$$
P_{e}=\frac{1}{Q} \sum_{q=1}^{Q}\left[\left.\sum_{K_{h}=0}^{K-1} P_{e}\right|_{K_{h}, q} \cdot P_{K_{h}}\left(K_{h}\right)\right]
$$

where $P_{K_{h}}\left(K_{h}\right)$ is the pdf of $K_{h}$ and given by (13), and $\left.P_{e}\right|_{K_{h}, q}$ is given by (B5) or (B8).

In case $K_{h}$ is small, the Gaussian approximation may be not accurate enough to study the BER performance. However, small values of $K_{h}$ appear with small hit probability and small conditional BER. Therefore, their contributions to the final average BER are not significant. In the numerical section, the results of Gaussian approximation are verified by Monte Carlo simulations, which reveal the applicability of the Gaussian approximation in various situations. The description of the simulation model is given in Appendix C.

\section{Numerical Results}

In our comparison, all systems have the same order of diversity $\left(D=L_{T}=4\right)$, the same processing gain $\left(N_{T}=S N=\right.$ $512)$, and the same total average received energy $\left(E_{b}=D \cdot E_{c}\right)$. Pseudonoise (PN) sequences applied in Monte Carlo simulations are Gold sequences of period 127, which are obtained from multiplying two primitive polynomials

$$
h_{1}(x)=x^{7}+x^{3}+x^{2}+x+1 \quad \text { and } \quad h_{2}(x)=x^{7}+x^{3}+1
$$

respectively. The initial loadings are given in [12] to generate a class of codes known as auto-optimal with least sidelobe energy (AO/LSE).

To find the optimum channel division, the average asymptotic (without AWGN and narrow-band interference) SNR per diversity branch $E\left[\gamma_{m l}^{(v)}\right]$ is plotted for different subchannel numbers $(S)$ in Fig. 4. From (17) one obtains that

$$
E\left[\gamma_{m l}^{(v)}\right]=\frac{3 N}{2(K-1) / Q \cdot L}= \begin{cases}\frac{3 N_{T}}{2(K-1) L_{T}} Q, & S \leq L_{T} \\ \frac{3 N_{T}}{2(K-1)} \frac{1}{M}, & S>L_{T} .\end{cases}
$$

For the MC DS system ( $M=S, Q=1)$, the SNR per diversity branch remains constant as long as $S \leq L_{T}$, but decreases after $S$ exceeds $L_{T}$. For the hybrid DS/SFH system, the SNR increases with $S$ before $S$ reaches $L_{T}$, and remains constant afterwards. The SNRs of the MC DS/SFH systems fall between those of the MC DS and the hybrid DS/SFH systems. It can be seen from Fig. 4 that the average asymptotic SNRs per diversity branch of all systems reach their highest when $S=L_{T}$. Since 


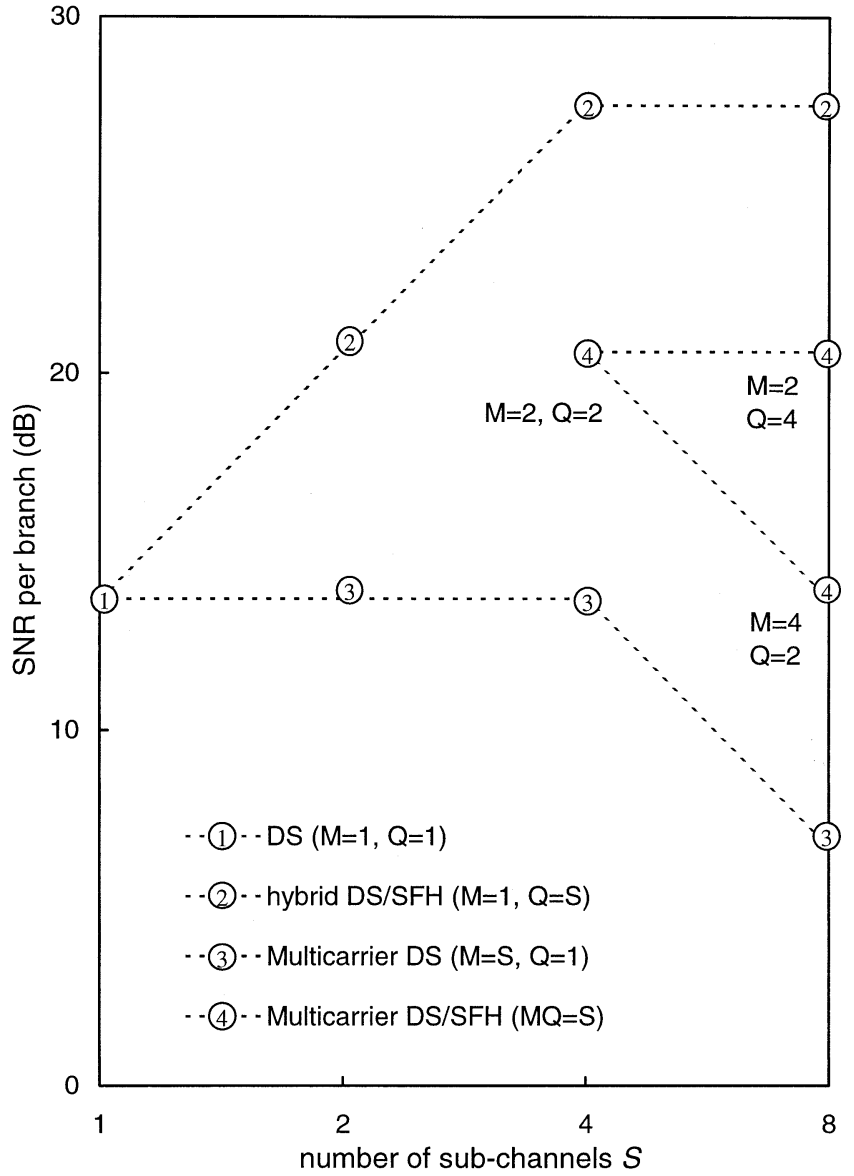

Fig. 4. Asymptotic SNR per branch with different number of subchannels $S$ $\left(K=50, N_{T}=512\right)$.

the conclusion above is not dependent on the values of $K$, it applies for all system loads.

In the following part, we set $S=L_{T}=4$ and consider three systems: the MC DS/SFH ( $M=2, Q=2)$, the MC DS ( $M=4, Q=1)$ systems, and the hybrid DS/SFH system $(M=1, Q=4)$. The number of antennas $V$ is chosen to be equal to $Q$ so that all systems have a diversity order of four. The BER performance of the three systems is compared along with that of the pure DS system $(S=1)$.

In the absence of narrow-band interference, $\left.\bar{\gamma}_{m}\right|_{K_{h}, q}$ in (B3) are identical for all $m$. Therefore, $\left.P_{e}\right|_{K_{h}, q}$ is given by (B5)

$$
\left.P_{e}\right|_{K_{h}, q}=\mu^{4} \sum_{i=0}^{3}\left(\begin{array}{c}
3+i \\
i
\end{array}\right)(1-\mu)^{i}
$$

where $\mu=\left[1-\sqrt{r_{0} /\left(1+r_{0}\right)}\right] / 2$, while $r_{0}$ is given by (B2) with $w=0$

$$
r_{0}=\left[2 K_{h} L /(3 N)+4\left(E_{b} / N_{0}\right)^{-1}\right]^{-1}
$$

where $L=4$ for pure DS system and $L=1$ for other systems.

The BER of all the systems with respect to $E_{b} / N_{0}$ in the absence of narrow-band interference is shown in Fig. 5, obtained by both Gaussian approximation and Monte Carlo simulation. The Gaussian approximation is shown to be a little optimistic in comparison with the simulation results when $E_{b} / N_{0}$ is large. However, the differences are not significant. It is also shown that the Gaussian approximation is more accurate for systems with

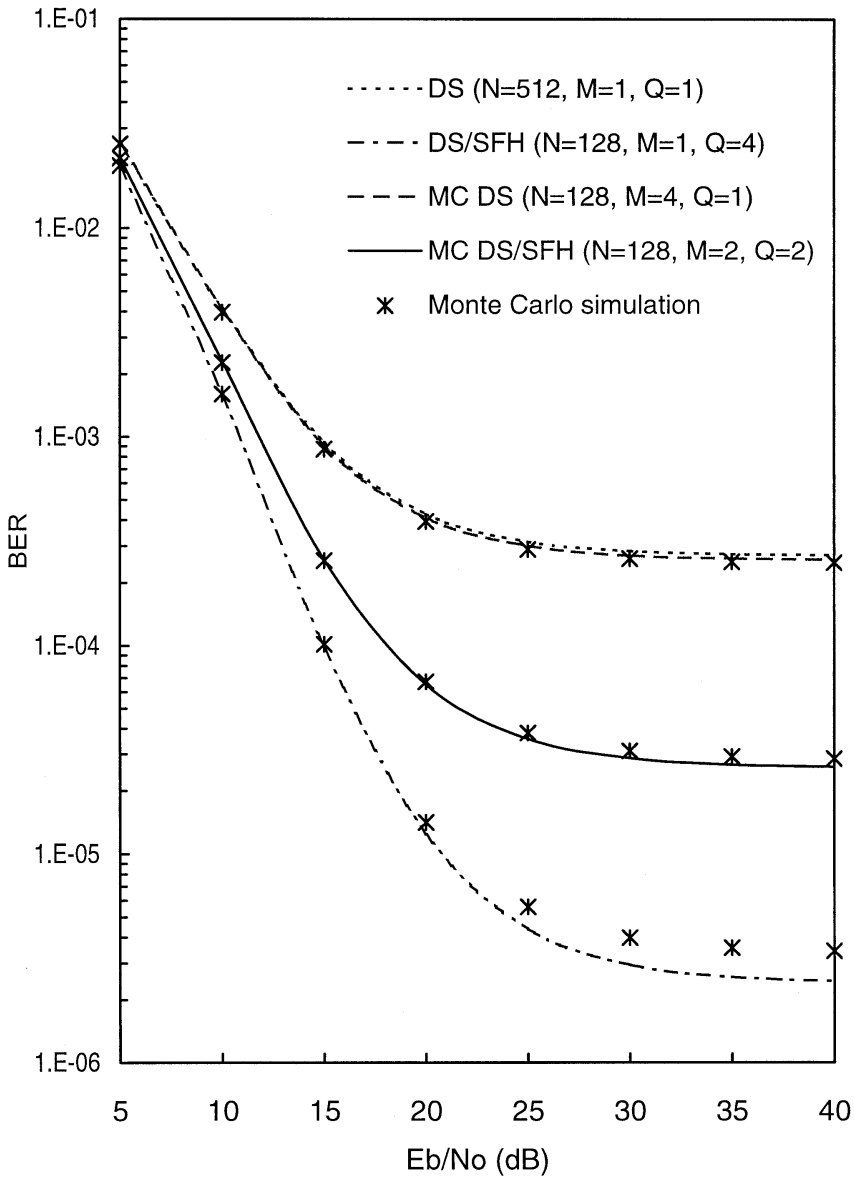

Fig. 5. BER performance with respect to $E_{b} / N_{0}$ in the absence of narrow-band interference $(K=50, w=-\infty \mathrm{dB})$.

smaller $Q$ which have a larger average hits number $E\left[K_{h}\right]$. It is also seen from Fig. 5 that the systems with larger $Q$ have better performance in the AWGN channel. It has already been shown in Fig. 4 that the systems with larger $Q$ have a higher SNR per diversity branch, therefore, with the same diversity order, the hybrid DS/SFH system has the best performance. The MC DS/SFH system, which employs fewer antennas and a shorter hopping pattern and, consequently, has lower implementation complexity, is second in performance. The MC DS and the pure DS systems exhibit almost the same performance since they have almost identical SNR per diversity branch.

The system performance in the CDMA overlay situations is of more concern in this paper. For single-carrier systems, namely, the hybrid DS/SFH and the pure DS systems, $\left.\bar{\gamma}_{m}\right|_{K_{h}, q}$ are still identical because at any instant the desired signal only use one subchannel. Therefore, $\left.P_{e}\right|_{K_{h}, q}$ is still given by (24). For the hybrid DS/SFH system, $r_{0}$ should be replaced by $r_{2}$ given by (B2) with $m=0$

$$
r_{2}=\left[2 K_{h} /(3 N)+4 \Delta(q) w \hat{\sigma}_{J}^{2} / N+4\left(E_{b} / N_{0}\right)^{-1}\right]^{-1} \text {. }
$$

For a pure DS system, $r_{0}$ should be replaced by $r_{3}$ given by

$$
r_{3}=\left[2 K_{h} L_{T} /(3 N)+4 w \bar{\sigma}_{J}^{2} / N+4\left(E_{b} / N_{0}\right)^{-1}\right]^{-1}
$$

where $\bar{\sigma}_{J}^{2}$ is given by

$$
\bar{\sigma}_{J}^{2}=2 \int_{0}^{1} \operatorname{sinc}(2 \pi p \tau)(1-\tau)(1-\tau / N) d \tau .
$$




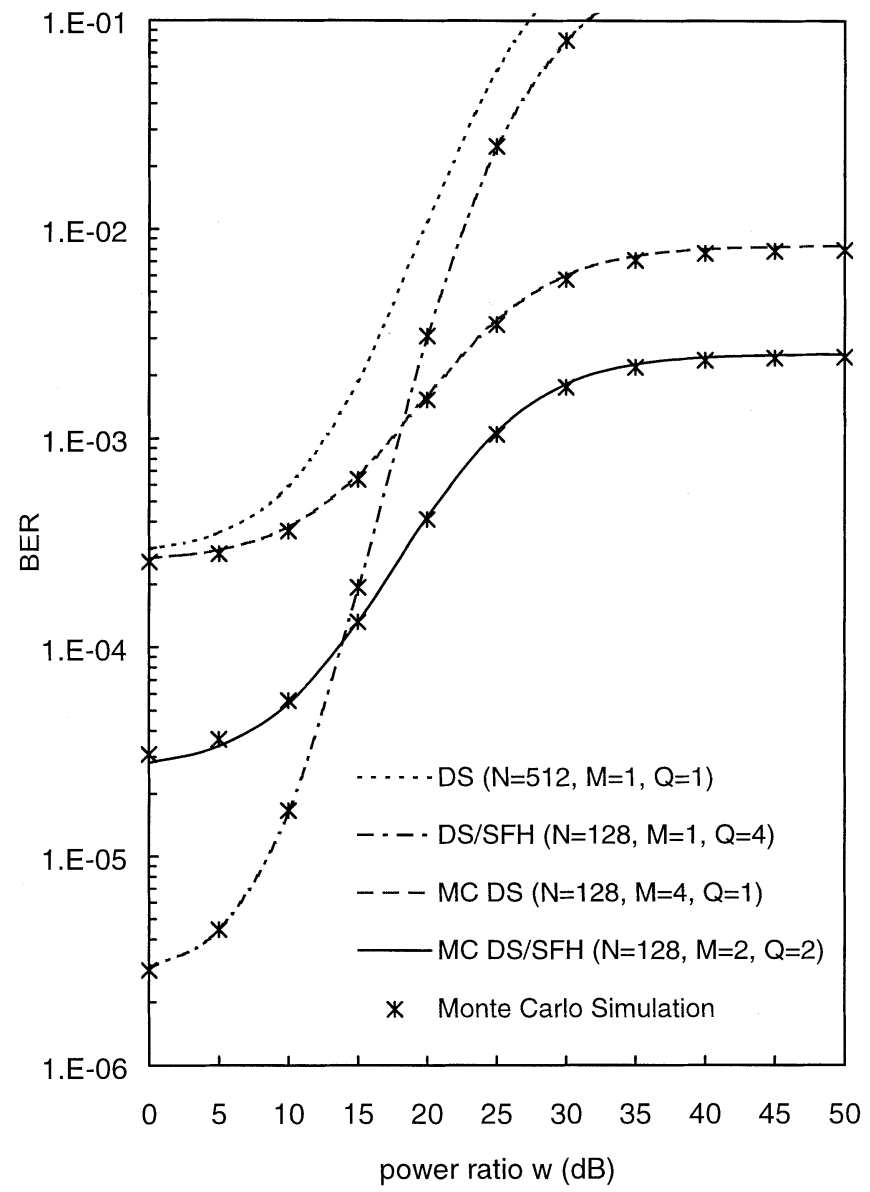

Fig. 6. BER performance versus interference power to signal power ratio $w$ $\left(K=50, B_{J}=B_{s}, E_{b} / N_{0} \rightarrow \infty\right)$.

For the multiple-carrier systems, $\left.\bar{\gamma}_{m}\right|_{K_{h}, q}$ are no longer identical for all $m$. For the MC DS system, the two middle subchannels are overlaid by the interference. The SNRs of the outputs from those two subchannels are identical and given by

$$
r_{1}=\left\{2 K_{h} L /(3 N)+4\left[w \hat{\sigma}_{J}^{2} / N+\left(E_{b} / N_{0}\right)^{-1}\right]\right\}^{-1}
$$

while the SNRs of the outputs from the other two subchannels free of interference are equal to $r_{0}$. Since the MC DS/SFH system uses one affected and one clean subchannel at any instant, and employs two antennas, it also has two affected diversity branches and two clean ones. Therefore, $\left.P_{e}\right|_{K_{h}, q}$ is identical for the two systems and given by (B10)

$$
\begin{aligned}
\left.P_{e}\right|_{K_{h}, q}=A_{10}^{2} \mu_{0}^{2}(3- & \left.2 \mu_{0}\right)+2 A_{10}^{2} A_{01} \mu_{0} \\
& +2 A_{01}^{2} A_{10} \mu_{1}+A_{01}^{2} \mu_{1}^{2}\left(3-2 \mu_{1}\right)
\end{aligned}
$$

where $A_{i j}=r_{j} /\left(r_{j}-r_{i}\right), \mu_{i}=\left(1-\sqrt{r_{i} /\left(1+r_{i}\right)}\right) / 2$, and $r_{0}$ and $r_{1}$ are given by (25) and (29), respectively.

In Fig. 6, the BER performance is plotted versus the interference power to signal power ratio $w$, while the interference bandwidth $B_{J}=B_{s}$. It is shown that the BERs of the pure DS and hybrid DS/SFH systems rises drastically with the interference power. Therefore, they are not suitable candidates for CDMA overlay. The MC systems have certain advantages because they can make use of their clean diversity branches and keep the BER at a lower level. Their performance as $w$ approaches infinity is no worse than that only the unaffected diversity branches are

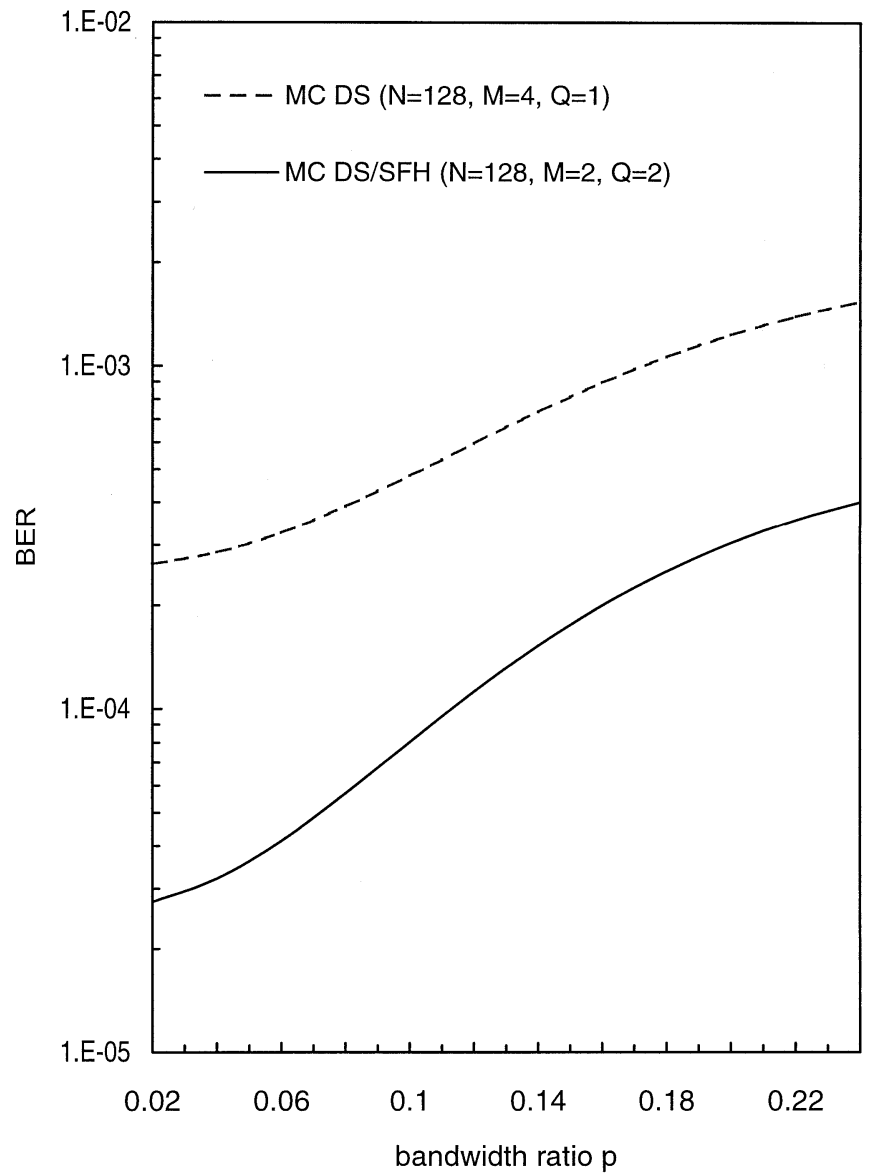

Fig. 7. BER performance versus interference bandwidth to system bandwidth ratio $p\left(K=50, w=20 \mathrm{~dB}, E_{b} / N_{0} \rightarrow \infty\right)$.

used. It is also seen that the MC DS/SFH system outperforms the MC DS system for any interference power. From (30), one can see that given $K_{h}$, the two systems have the same BER. However, the MC DS/SFH system has a larger $Q$ that results in a smaller mean value of $K_{h}$ and, therefore, a higher average SNR per diversity branch. In general, the MC DS/SFH system can suppress the narrow-band interference as effectively as the MC DS system while undergoing lower MAI.

Fig. 7 illustrates the effects of the interference bandwidth on the performance of the MC DS/SFH and the MC DS systems $\left(K=50, w=20 \mathrm{~dB}, E_{b} / N_{0} \rightarrow \infty\right)$. It is seen that the performance of both systems degrades as the interference bandwidth increases, but the MC DS/SFH system keeps its advantages. The BERs of both systems for different system loads $(K)$ are given in Fig. $8\left(w=20 \mathrm{~dB}, B_{J}=B_{s}, E_{b} / N_{0} \rightarrow \infty\right)$, which shows that the advantages of the MC DS/SFH system become more noticeable with heavy system loads.

Finally, we consider the cases that the narrow-band interference is not located in the center of the system bandwidth. The performance of the MC DS/SFH and the MC DS systems with respect to the location of the interference central frequency $f_{J}$ $\left(B_{J}=B_{s}, w=50 \mathrm{~dB}\right)$ is shown in Fig. 9. It is seen that both systems perform best when the interference is limited within one subchannel $\left(f_{J}=f_{s}, s=1, \ldots, 4\right)$ because more clean subchannels are available, especially for the MC DS system. In those situations, the MC DS system has three unaffected di- 


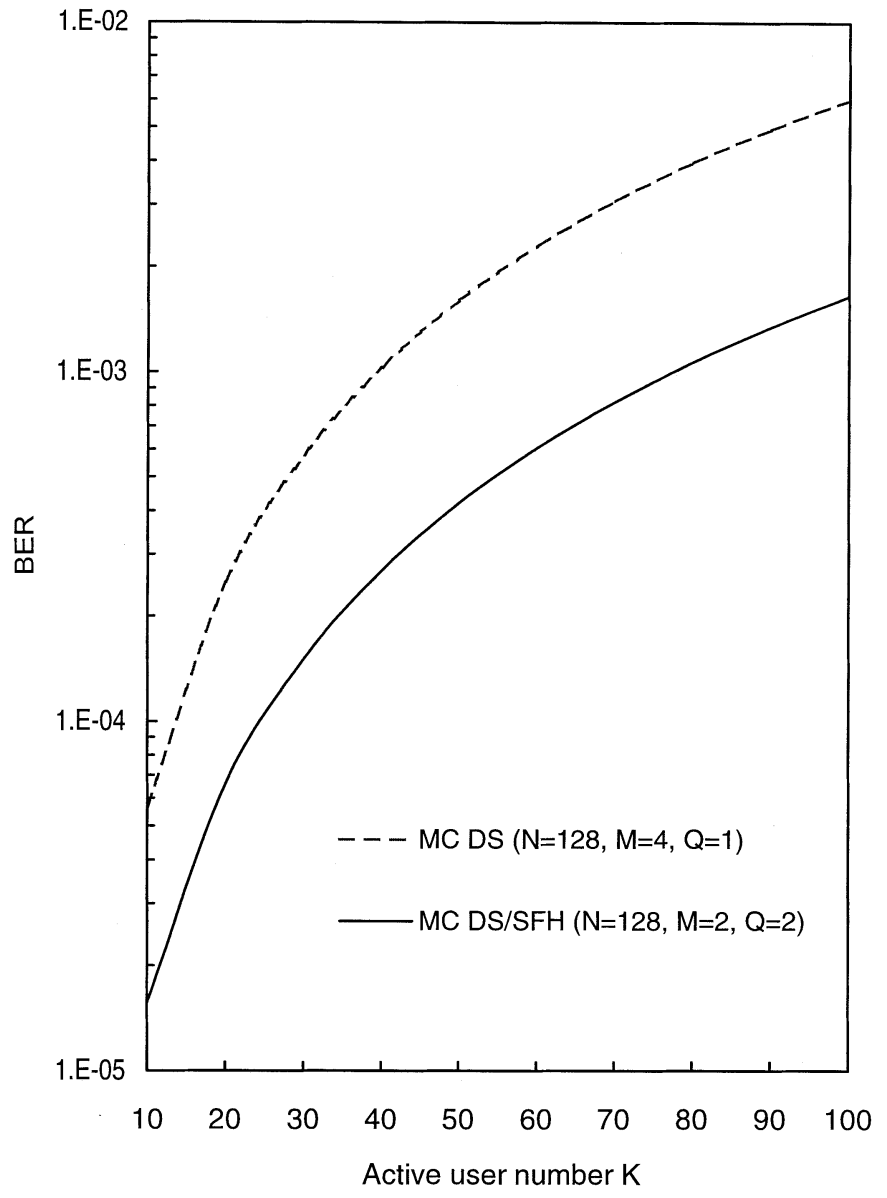

Fig. 8. BER performance versus active user number $K\left(w=20 \mathrm{~dB}, B_{J}=\right.$ $\left.B_{s}, E_{b} / N_{0} \rightarrow \infty\right)$.

versity branches while the MC DS/SFH system has only two in the worst case. That explains why the performance of the MC DS system is better when active user number is small (e.g., $K=10$ ). However, when user number is large so that MAI is comparable to the narrow-band interference, the MC DS/SFH still performs better.

\section{CONCLUding REMARKS}

The MC DS/SFH-CDMA systems are investigated in a multipath Rayleigh-fading channel, with or without narrow-band interference. The performance is compared with that of the MC DS system, along with the hybrid DS/SFH system and the pure DS system. The results show that in a multipath channel, it is advisable to divide the total bandwidth into subchannels with a mutual separation of the channel coherence bandwidth, and use frequency and antenna diversity instead of multipath diversity. Though MC DS system can suppress the narrow-band interference effectively via frequency diversity, it does not help to reduce the MAI. The MC DS/SFH system, with an appropriate combination of $\mathrm{MC}$ and $\mathrm{SFH}$ techniques, can provide sufficient frequency diversity and reduce MAI simultaneously, and as a result, achieves better performance.

Note that the performance comparison of the MC DS/SFH with conventional MC CDMA is made under the condition of the same diversity order. Using multiple antennas most likely

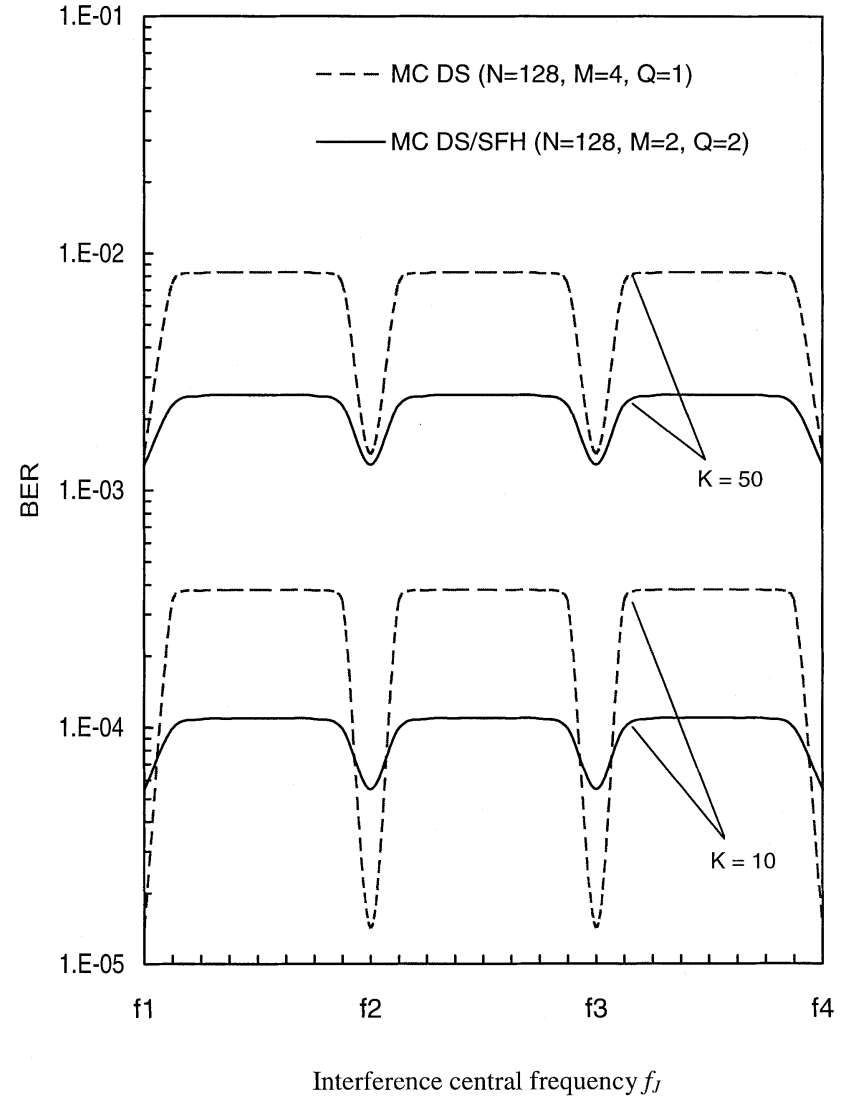

Fig. 9. BER with respect to interference central frequency $f_{J}(w=50 \mathrm{~dB}$, $\left.B_{J}=B_{s}, E_{b} / N_{0} \rightarrow \infty\right)$.

in base stations to obtain diversity in the MC DS/SFH-CDMA system may seem to be unfair to other systems. However, the MC DS-CDMA system employs multiple carriers to achieve frequency diversity. In terms of the number of correlators and gain estimators required in the receiver, the proposed system is no more complicated than the others. We admit that antenna diversity may cost more in implementation, but it provides better performance.

\section{APPENDIX A}

THE DERIVATION OF $\sigma_{J}^{2}\left(s_{m}\right)$

Substituting $J^{\left(s_{m}, v\right)}(t)$ in (11) by (6a) with $s=s_{m}$ and neglecting the high-frequency terms, we have

$$
J_{m l}^{(v)}=\int_{\lambda T}^{(\lambda+1) T} \hat{J}^{\left(s_{m}, v\right)}\left[t-(L-1-l) T_{c}\right] a_{i}(t) d t
$$

where

$$
\begin{aligned}
\hat{J}^{\left(s_{m}, v\right)}(t) & =\Delta\left(s_{m}\right) \operatorname{Re}\left\{\left[J_{I}^{(v)}(t)+j J_{Q}^{(v)}(t)\right]\right. \\
\cdot & \left.\exp \left[ \pm j 2 \pi\left(B_{s} / 2-B_{J} / 4\right) t-j \Omega_{i, l}^{\left(s_{m}, v\right)}\right]\right\}
\end{aligned}
$$

where

$\Omega_{i, l}^{\left(s_{m}, v\right)}=\Phi_{i, l}^{\left(s_{m}, v\right)}+2 \pi\left[m B_{H} \pm\left(B_{s} / 2-B_{J} / 4\right)\right](L-1-l) T_{c}$.

The autocorrelation function of $\hat{J}^{\left(s_{m}, v\right)}(t)$ is given by (7) with $f_{s}=0$, i.e.,

$\hat{R}_{J}(\tau)$

$=\Delta\left(s_{m}\right) P_{J} / 2 \operatorname{sinc}\left(\pi B_{J} \tau / 2\right) \cos \left[2 \pi\left(B_{s} / 2-B_{J} / 4\right) \tau\right]$. 
Therefore, the variance of $J_{m l}^{(v)}$, conditioned on $s_{m}$, is given by

$$
\begin{aligned}
\sigma_{J}^{2}\left(s_{m}\right)= & \int_{\lambda T}^{(\lambda+1) T} \int_{\lambda T}^{(\lambda+1) T} E\left\{\hat{J}^{\left(s_{m}, v\right)}\left[t_{1}-(L-1-l) T_{c}\right]\right. \\
& \left.\cdot \hat{J}^{\left(s_{m}, v\right)}\left[t_{2}-(L-1-l) T_{c}\right]\right\} \\
& \cdot E\left[a_{i}\left(t_{1}\right) a_{i}\left(t_{2}\right)\right] d t_{1} d t_{2} \\
= & \int_{\lambda T}^{(\lambda+1) T} \int_{\lambda T}^{(\lambda+1) T} \hat{R}_{J}\left(t_{1}-t_{2}\right) R_{i}\left(t_{1}-t_{2}\right) d t_{1} d t_{2} \\
= & \int_{-T}^{T} \hat{R}_{J}(\tau) R_{i}(\tau)(T-|\tau|) d \tau
\end{aligned}
$$

where $R_{i}(\tau)$ is the autocorrelation function of $a_{i}(t)$ and triangular in $\left(-T_{c}, T_{c}\right)$. Substituting $\hat{R}_{J}(\tau)$ in (A4) by (A3), one obtains

$$
\begin{aligned}
\sigma_{J}^{2}\left(s_{m}\right)= & \Delta\left(s_{m}\right) P_{J} \int_{0}^{T_{c}} \sin c\left(\pi B_{J} \tau / 2\right) \\
& \cdot \cos \left[2 \pi\left(B_{s} / 2-B_{J} / 4\right) \tau\right]\left(1-\tau / T_{c}\right)(T-\tau) d \tau \\
= & \Delta\left(s_{m}\right) \frac{P_{J} T^{2}}{N} \hat{\sigma}_{J}^{2}
\end{aligned}
$$

where

$\hat{\sigma}_{J}^{2}=\int_{0}^{1} \sin c(\pi p S \tau) \cos [2 \pi(1-p S / 2) \tau](1-\tau)(1-\tau / N) d \tau$

where $p$ is given by (4) and $S=M Q$.

\section{APPENDIX B}

THE DeRIVATION OF $\left.P_{e}\right|_{K_{h}, q}$

Since $\beta_{i l}^{\left(s_{m}, v\right)}$ is Rayleigh distributed, the SNR $\left.\gamma_{m l}^{(v)}\right|_{\beta_{i l}^{\left(s_{m}, v\right)}, K_{h}, q}$ in (17) has a chi-square distribution, conditioned on $K_{h}$ and $q$. Its characteristic function is given by

$$
\psi_{m l}^{(v)}(j u)=\frac{1}{1-\left.j u \bar{\gamma}_{m}\right|_{K_{h}, q}}
$$

where $\left.\bar{\gamma}_{m}\right|_{K_{h}, q}$ is the mean of $\left.\gamma_{m l}^{(v)}\right|_{\beta_{i l}^{\left(s_{m}, v\right)}, K_{h}, q}$ over $\beta_{i l}^{\left(s_{m}, v\right)}$ and given by

$$
\begin{aligned}
\left.\bar{\gamma}_{m}\right|_{K_{h}, q}= & E_{\beta}\left[\left.\gamma_{m l}^{(v)}\right|_{\beta_{i l}^{\left(s_{m}, v\right)}, K_{h}, q}\right] \\
= & \left\{2 K_{h} L /(3 N)+D\left[\Delta(q+m Q) w \hat{\sigma}_{J}^{2} / N\right.\right. \\
& \left.\left.+\left(E_{b} / N_{0}\right)^{-1}\right]\right\}^{-1}
\end{aligned}
$$

Because $\left\{\beta_{i l}^{\left(s_{m}, v\right)}\right\}$ are mutually independent, the characteristic function of $\left.\gamma_{b}\right|_{\left\{\beta_{i l}^{\left(s_{m}, v\right)}\right\}, K_{h}, q}$ is simply the product of all $\psi_{m l}^{(v)}(j u)$, i.e.,

$$
\begin{aligned}
\psi_{b}(j u) & =\prod_{v=1}^{V} \prod_{m=0}^{M-1} \prod_{l=0}^{L-1} \psi_{m l}^{(v)}(j u) \\
& =\prod_{m=0}^{M-1} \frac{1}{\left(1-\left.j u \bar{\gamma}_{m}\right|_{K_{h}, q}\right)^{V L}} .
\end{aligned}
$$

The pdf of $\gamma_{b}, f_{\gamma}\left(\gamma_{b} \mid K_{h}, q\right)$, conditioned on $K_{h}$ and $q$, can be obtained by inverse Fourier transform of $\psi_{b}(j u)$, and $\left.P_{e}\right|_{\left(K_{h}, q\right)}$ is given by

$$
\left.P_{e}\right|_{K_{h}, q}=\int_{0}^{\infty} P_{e}\left(\gamma_{b}\right) f_{\gamma}\left(\gamma_{b} \mid K_{h}, q\right) d \gamma_{b} .
$$

When all diversity branches have the same average SNR equal to $r$, i.e., $\psi_{b}(j u)=1 /(1-j u r)^{D}$, where $D$ is the diversity order, $\left.P_{e}\right|_{K_{h}, q}$ has a closed form solution [10]

$$
\left.P_{e}\right|_{K_{h}, q}=P(D, r)=\mu^{D} \sum_{i=0}^{D-1}\left(\begin{array}{c}
D-1+i \\
i
\end{array}\right)(1-\mu)^{i}
$$

where $\mu=[1-\sqrt{r /(1+r)}] / 2$.

When the SNRs of different branches are not identical (in the presence of narrow-band interference), $\psi_{b}(j u)$ can be expressed by a partial fraction expansion, i.e.,

$$
\psi_{b}(j u)=\frac{1}{\prod_{i}\left(1-j u r_{i}\right)^{D_{i}}}=\sum_{i} \sum_{j=1}^{D_{i}} \frac{B_{i, j}}{\left(1-j u r_{i}\right)^{j}}
$$

where $\sum_{i} D_{i}=D$ and

$$
B_{i, j}=\left.\frac{\left(-r_{i}\right)^{\left(j-D_{i}\right)}}{\left(D_{i}-j\right) !}\left\{\frac{d^{\left(D_{i}-j\right)}}{d x^{\left(D_{i}-j\right)}}\left[\left(1-r_{i} x\right)^{D_{i}} \psi_{b}(x)\right]\right\}\right|_{x=1 / r_{i}}
$$

where $\left(d^{k} / d x^{k}\right)[f(x)]$ represents the $k$ th derivative of $f(x)$. Since the inverse Fourier transform and integration are linear, the corresponding probability of bit error can be written as

$$
\left.P_{e}\right|_{K_{h}, q}=\sum_{i} \sum_{d=1}^{D_{i}} B_{i, d} P\left(d, r_{i}\right) \text {. }
$$

For example, when $D=4$, two branches have SNR $r_{0}$ and the other two branches have SNR $r_{1}$ (the case we meet in the numerical section)

$$
\psi_{b}(j u)=\frac{1}{\left(1-j u r_{0}\right)^{2}\left(1-j u r_{1}\right)^{2}}
$$

one has

$$
\begin{aligned}
\left.P_{e}\right|_{K_{h}, q}= & A_{10}^{2} P\left(2, r_{0}\right)+2 A_{10}^{2} A_{01} P\left(1, r_{0}\right) \\
& +2 A_{01}^{2} A_{10} P\left(1, r_{1}\right)+A_{01}^{2} P\left(2, r_{1}\right) \\
= & A_{10}^{2} \mu_{0}^{2}\left(3-2 \mu_{0}\right)+2 A_{10}^{2} A_{01} \mu_{0} \\
& +2 A_{01}^{2} A_{10} \mu_{1}+A_{01}^{2} \mu_{1}^{2}\left(3-2 \mu_{1}\right)
\end{aligned}
$$

where $A_{i j}=r_{j} /\left(r_{j}-r_{i}\right)(i \neq j, r i \neq r j)$ and $\mu_{i}=[1-$ $\left.\sqrt{r_{i} /\left(1+r_{i}\right)}\right] / 2$.

\section{APPENDIX C \\ The Monte Carlo Simulation Model}

For rectangular chips and $0 \leq n T_{c} \leq \tau \leq(n+1) T_{c} \leq T$, as shown in [11], the partial cross-correlation functions $R_{k i}(\tau)$ and $\hat{R}_{k i}(\tau)$ are given by

$$
\begin{aligned}
R_{k i}(\tau)= & C_{k i}(n-N) T_{c}+\left[C_{k i}(n+1-N)-C_{k i}(n-N)\right] \\
& \cdot\left(\tau-n T_{c}\right) \\
\hat{R}_{k i}(\tau)= & C_{k i}(n) T_{c}+\left[C_{k i}(n+1)-C_{k i}(n)\right]\left(\tau-n T_{c}\right)(\mathrm{C} 1)
\end{aligned}
$$

where the discrete aperiodic cross-correlation term $C_{k i}(\cdot)$ is defined as

$$
C_{k i}(n)= \begin{cases}\sum_{j=0}^{N-1-n} a_{k}^{(j)} a_{i}^{(j+n)}, & 0 \leq n \leq N-1 \\ \sum_{j=0}^{N-1+n} a_{k}^{(j-n)} a_{i}^{(j)}, & -(N-1) \leq n \leq 0 \\ 0, & \text { else. }\end{cases}
$$


Once the code sequences are obtained, $R_{k i}(\tau)$ and $\hat{R}_{k i}(\tau)$ are determined by $\tau$. Note that $I_{k l^{\prime}}^{(m, v)}$ in (14) is a zero-mean Gaussian random variable if $\tau_{k}$ is given, which follows from the fact that

$$
\beta_{k l^{\prime}}^{\left(s_{m}, v\right)} \cos \left[\Phi_{k l^{\prime}}^{\left(s_{m}, v\right)}-\Phi_{i l}^{\left(s_{m}, v\right)}\right]
$$

is a zero-mean Gaussian variable with variance $\rho$, therefore,

$$
b_{k}^{(\lambda-1)} \beta_{k l^{\prime}}^{\left(s_{m}, v\right)} \cos \left[\Phi_{k l^{\prime}}^{\left(s_{m}, v\right)}-\Phi_{i l}^{\left(s_{m}, v\right)}\right]
$$

and

$$
b_{k}^{(\lambda)} \beta_{k l^{\prime}}^{\left(s_{m}, v\right)} \cos \left[\Phi_{k l^{\prime}}^{\left(s_{m}, v\right)}-\Phi_{i l}^{\left(s_{m}, v\right)}\right]
$$

are also zero-mean Gaussian variables with the same variance. The conditional variance of $I_{k l^{\prime}}^{(m, v)}$ is given by

$$
\sigma_{k l^{\prime}}^{2}=2 \rho P\left[R_{k i}^{2}\left(\Gamma_{l^{\prime} l}^{k}\right)+\hat{R}_{k i}^{2}\left(\Gamma_{l^{\prime} l}^{k}\right)\right] .
$$

It is also obvious that $\left\{I_{k l^{\prime}}^{(m, v)}\right\}$ are all uncorrelated since

$$
E\left\{\cos \left[\Phi_{k_{1} l_{1}}^{\left(s_{m}, v\right)}-\Phi_{k_{2} l_{2}}^{\left(s_{m}, v\right)}\right]\right\}=0, \quad \text { for } k_{1} \neq k_{2} \text { or } l_{1} \neq l_{2} \text {. }
$$

Therefore, conditioned on $b_{i}^{(\lambda)}, \beta_{i l}^{\left(s_{m}, v\right)},\left\{\tau_{k}\right\}, K_{h}$, and $q$, $\xi_{m l}^{(v)}(\lambda)$ is a Gaussian random variable. The SNR of $\xi_{m l}^{(v)}(\lambda)$ is defined as

$$
\begin{aligned}
\left.\gamma_{m l}^{(v)}\right|_{\beta_{i l}^{\left(s_{m}, v\right)},\left\{\tau_{k}\right\}, K_{h}, q} & \frac{E^{2}\left[\xi_{m l}^{(v)}(\lambda) \mid \beta_{i l}^{\left(s_{m}, v\right)}\right]}{2 \operatorname{Var}\left[\xi_{m l}^{(v)}(\lambda) \mid\left\{\tau_{k}\right\}, K_{h}, q\right]} \\
= & \frac{E_{c} T\left(\beta_{i l}^{\left(s_{m}, v\right)}\right)^{2}}{2 \rho} \\
& \cdot\left[\sum_{k=1}^{K_{h}} \sum_{l^{\prime}=0}^{L-1} \sigma_{k l^{\prime}}^{2}+\sigma_{J}^{2}(q+m Q)+N_{0} T\right]^{-1} .
\end{aligned}
$$

The instantaneous SNR of $\xi(\lambda)$, conditioned on $\left\{\beta_{i l}^{\left(s_{m}, v\right)}\right\}$, $\left\{\tau_{k}\right\}, K_{h}$, and $q$ is given by

$$
\begin{aligned}
\left.\gamma_{b}\right|_{\left.\beta_{i l}^{\left(s_{m}\right.}, v\right)},\left\{\tau_{k}\right\}, K_{h}, q \\
=\left.\sum_{v=1}^{V} \sum_{m=0}^{M-1} \sum_{l=0}^{L-1} \gamma_{m l}^{(v)}\right|_{\beta_{i l}^{\left(s_{m}, v\right)},\left\{\tau_{k}\right\}, K_{h}, q} \\
=\sum_{v=1}^{V} \sum_{m=0}^{M-1} \sum_{l=0}^{L-1} \frac{\left(\beta_{i l}^{\left(s_{m} v\right)}\right)^{2}}{2 \rho} \\
\quad \cdot\left\{T^{-2} \sum_{k=1}^{K_{h}} \sum_{l^{\prime}=1}^{L-1}\left[R_{k i}^{2}\left(\Gamma_{l^{\prime} l}^{k}\right)+\hat{R}_{k i}^{2}\left(\Gamma_{l^{\prime} l}^{k}\right)\right]\right. \\
\left.\quad+D\left[\Delta(q+m Q) w \hat{\sigma}_{J}^{2} / N+\left(E_{b} / N_{0}\right)^{-1}\right]\right\}^{-1} .
\end{aligned}
$$

To obtain the average BER $P_{e}$, the conditional BER is averaged over the statistics of $\gamma_{b}$, that is

$$
P_{e}=\int_{0}^{\infty} P_{e}\left(\gamma_{b}\right) f_{\gamma}\left(\gamma_{b}\right) d \gamma_{b}
$$

where $f_{\gamma}\left(\gamma_{b}\right)$ represents the pdf of $\gamma_{b}$. It is difficult to get the analytical results, therefore Monte Carlo integration over $\left\{\beta_{i l}^{\left(s_{m}, v\right)}\right\},\left\{\tau_{k}\right\}, K_{h}$, and $q$ is performed to get $P_{e}$.

\section{REFERENCES}

[1] J. Wang and L. B. Milstein, "CDMA overlay situations for microcellular mobile communications," IEEE Trans. Commun., vol. 43, pp. 603-614, Feb. 1995.

[2] B. J. Rainbolt and S. L. Miller, "The necessity for and use of CDMA transmitter filtering in overlay systems," IEEE J. Select. Areas Commun., vol. 16, pp. 1756-1764, Dec. 1998.

[3] S. Kondo and L. B. Milstein, "Performance of multicarrier DS CDMA systems," IEEE Trans. Commun., vol. 44, pp. 238-246, Feb. 1996.

[4] T. M. Lok, T. F. Wong, and J. S. Lehnert, "Blind adaptive signal reception for MC-CDMA systems in Rayleigh fading channels," IEEE Trans. Commun., vol. 47, pp. 464-471, Mar. 1999.

[5] E. A. Sourour and M. Nakagawa, "Performance of orthogonal multicarrier CDMA in a multipath fading channel," IEEE Trans. Commun., vol. 44, pp. 356-367, Mar. 1996.

[6] Q. Chen, E. S. Sousa, and S. Pasupathy, "Multicarrier CDMA with adaptive frequency hopping for mobile radio systems," IEEE J. Select. Areas Commun., vol. 14, pp. 1852-1858, Dec. 1996.

[7] D. Lee and L. B. Milstein, "Comparison of multicarrier DS CDMA broadcast systems in a multipath fading channel," IEEE Trans. Commun., vol. 47, pp. 1897-1904, Dec. 1999.

[8] J. Wang and M. Moeneclaey, "Hybrid DS/SFH-SSMA with predetection diversity and coding for indoor radio multipath Rician fading channels," IEEE Trans. Commun., vol. 40, pp. 1654-1662, Oct. 1992.

[9] B. Solaiman, A. Glavieux, and A. Hillion, "Equal gain diversity improvement in fast frequency hopping spread spectrum multiple-access (FFH-SSMA) communications over Rayleigh fading channels," IEEE J. Select. Areas Commun., vol. 7, pp. 140-147, Jan. 1989.

[10] J. G. Proakis, Digital Communication, 3rd ed. New York: McGrawHill, 1995.

[11] M. B. Pursley, "Performance evaluation for phase-coded spread spectrum multiple access communication-Part I: System analysis," IEEE Trans. Commun., vol. 25, pp. 795-799, Aug. 1977.

[12] H. F. A. Roefs and M. B. Pursley, "Correlation parameters of random sequences and maximal length sequences for spread-spectrum multiple-access communications," IEEE Trans. Commun., vol. COM-27, pp. 1797-1604, Oct. 1979

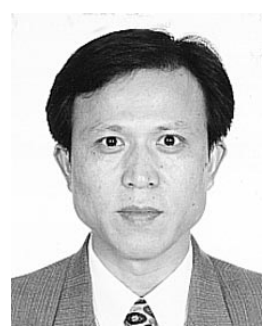

Jiangzhou Wang (M'91-SM'94) received the B.S. and M.S. degrees from Xidian University, Xian, China, in 1983 and 1985, respectively, and the Ph.D. degree (with Greatest Distinction) from the University of Ghent, Belgium, in 1990, all in electrical engineering.

From 1990 to 1992, he was a Postdoctoral Fellow at the University of California, San Diego, where he worked on research and development of cellular CDMA systems. From 1992 to 1995, he was a Senior System Engineer at Rockwell International Corporation, Newport Beach, CA, where he worked on the development and system design of wireless communications. Since 1995, he has been with the University of Hong Kong, where he is currently a Coordinator of Telecommunications Group and an Associate Professor. He has held a Visiting Professor position in NTT DoCoMo, Japan. He has published over 100 papers, including more than 20 IEEE TRANSACTIONS/JOURNAL papers in the areas of wireless mobile and spread-spectrum communications. He has written/edited two books, entitled Broadband Wireless Communications (Norwell, MA: Kluwer, 2001) and $3 G$ Mobile Enhanced Technologies (Norwood, MA: Artech House, 2002), respectively.

Dr. Wang is an Editor for IEEE TRANSACTIONS ON COMMUNICATIONS and a Guest Editor for IEEE JOURNAL ON SELECTED AREAS IN COMMUNICATIONS. He was a Technical Chairman of IEEE Workshop in 3G Mobile Communications, 2000. He holds one U.S. patent in the GSM system. He is listed in Who's Who in the World.

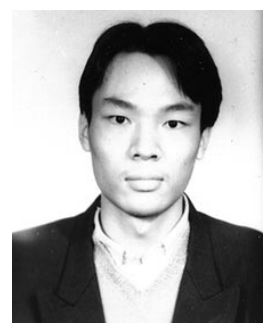

Hu Huang received the B.S. degree from the University of Science and Technology of China and the M.Phil. degree from the University of Hong Kong in 1997 and 2000, respectively, all in electrical engineering. Currently he is working towards the Ph.D. degree in the Electrical and Computer Engineering Department, University of Maryland, College Park. 